\title{
Implantatentfernung nach Osteosynthese beim alten Menschen
}

\author{
Jan Pützler, Richard Stange, Christoph Domnick, Michael Raschke
}

\section{Zusammenfassung}

Im Allgemeinen sind alte Patienten aufgrund schlechterer Knochenqualität, vorbestehenden Deformitäten, Degenerationen und oft kompromittierten Weichteilverhältnissen im Rahmen vaskulärer Vorerkrankungen eher gefährdet, eine perioperative Komplikation zu erleiden als jüngere Patienten. Die Indikation zur Implantatentfernung (IE) ist daher bei diesem Patientengut sehr streng zu stellen. Es zeigt sich jedoch in Deutschland in den vergangenen 10 Jahren ein Anstieg an IE bei älteren Patienten ( $\geq 65$ Jahren; +7\%), obwohl die Gesamtzahl der IE über alle Altersgruppen hinweg eher abgenommen hat (-7\%). Aufgrund einer Morbiditätskompression bestehen bei älteren Patienten immer seltener absolute Kontraindikationen gegen den operativen Eingriff. Es muss jedoch immer eine individuelle Risiko-Nut-
zen-Abwägung erfolgen und der Patient muss aktiv in den Entscheidungsprozess eingebunden werden. Die frühzeitige IE bei beschwerdefreien älteren Patienten, mit dem Ziel, einen möglichen späteren Eingriff zur Endoprothesenimplantation im Bereich von Knie, Hüfte und Schulter zu vereinfachen, ist aufgrund des Risikos des zusätzlichen Eingriffs nicht zu empfehlen. Ein einzeitiger Wechsel zu einer Endoprothese kann meist trotz lang einliegender Osteosynthesematerialien gut gelingen. Eine IE ist daher ausschließlich symptomatischen Patienten zu empfehlen.

\section{Implant Removal After Fracture Fixation in Elderly Patients}

Elderly patients ( $\geq 65$ years) are more likely to suffer from perioperative complications due to preconditions, including osteopenia, deformities, degeneration and often compromised soft tissue in the context of vascular diseases. Thus, the indication for implant removal must be very strict. However, in the last decade, the number of implant removals in Germany has risen in elderly patients $(+7 \%)$, while the number throughout all age groups has declined $(-7 \%)$. As a result of morbidity compression, there is frequently a lack of clear contraindications against surgery in elderly patients. Nevertheless, an individual risk-benefit analysis has to be made in every case and the patient has to take part in in the decision-making process. Early removal of an implant in asymptomatic elderly patients to facilitate possible future arthroplasty cannot be recommended, as there is an inherent risk of the additional surgical intervention. One-stage exchange from fracture fixation device to prosthesis can succeed in most cases. Therefore, implant removal can only be recommended to symptomatic patients.

\section{Einleitung}

Die Implantatentfernung (IE) zählt in Deutschland und weltweit immer noch mit zu den häufigsten elektiven Routineeingriffen am menschlichen Skelett [1, 2]. Nach Zahlen des statistischen Bundesamts wurden im Jahr 2013 in Deutschland insgesamt über $180000 \mathrm{IE}$ durchgeführt. Bezogen auf die spezifischen OPS-Codes für die Osteosynthesematerialien Draht, Schraube, Zuggurtung, Platte und Nagel waren es immerhin noch über 110000 IE. Erfasst wurden dabei ausschließlich stationäre Eingriffe, da im ambulanten Sektor keine Zahlen existieren. Die tatsächliche Anzahl wird daher noch um ein Vielfaches höher sein.

OP-JOURNAL 2016; 32: 106-113

(c) Georg Thieme Verlag KG Stuttgart · New York DOI http://dx.doi.org/10.1055/s-0042-116236
Im Allgemeinen wird die Indikation zur IE jedoch aus verschiedenen Gründen heute deutlich restriktiver gestellt als in den 1970er-Jahren. Die ursprünglich von der AO empfohlene Durchführung einer routinemäßigen IE nach Frakturheilung entstammt einer Zeit, in der Stahl das am häufigsten verwendete Osteosynthesematerial war [3]. Stahl stand im Verdacht, verschiedene gesundheitliche Beeinträchtigungen aufgrund von Korrosion zu verursachen (u.a. Allergien und Tumorgenese). In den folgenden Jahrzehnten zeigte sich, dass Allergien außerordentlich selten auftraten und sich eine Kanzerogenität wissenschaftlich nicht nachweisen ließ, sodass diese Phänomene schon seit den 1990er-Jahren nicht mehr als Indikation für eine routinemäßige IE angesehen wurden [4,5].

Für die Entfernung des Osteosynthesematerials nach Frakturheilung sprechen die in Tab. 1 genannten Gründe. Darüber hinaus zeigt sich im klinischen Alltag oft, dass nach dem Verständnis vieler Patienten der Heilungsverlauf und damit auch die Therapie erst mit der vollständigen IE abgeschlossen sind. Dieser Umstand zeigt, dass bereits zum Zeitpunkt der initialen OP eine Aufklärung des Patienten über den Nutzen einer IE und die Möglichkeit des Verbleibens des Implantats bei komplikationslosem Heilungsverlauf aufgeklärt werden sollte.

Im deutschsprachigen Raum hat Titan aufgrund seiner höheren zyklischen Belastbarkeit und besseren Biokompatibilität Stahlimplantate weitgehend verdrängt. Mit dem Einzug von Titanimplantaten ergaben sich jedoch auch neue Schwierigkeiten bei der IE. So ist die Oberflächenbeschaffenheit der Titanimplantate deutlich stärker von Osseointegration und knöcherner Überbauung be- 

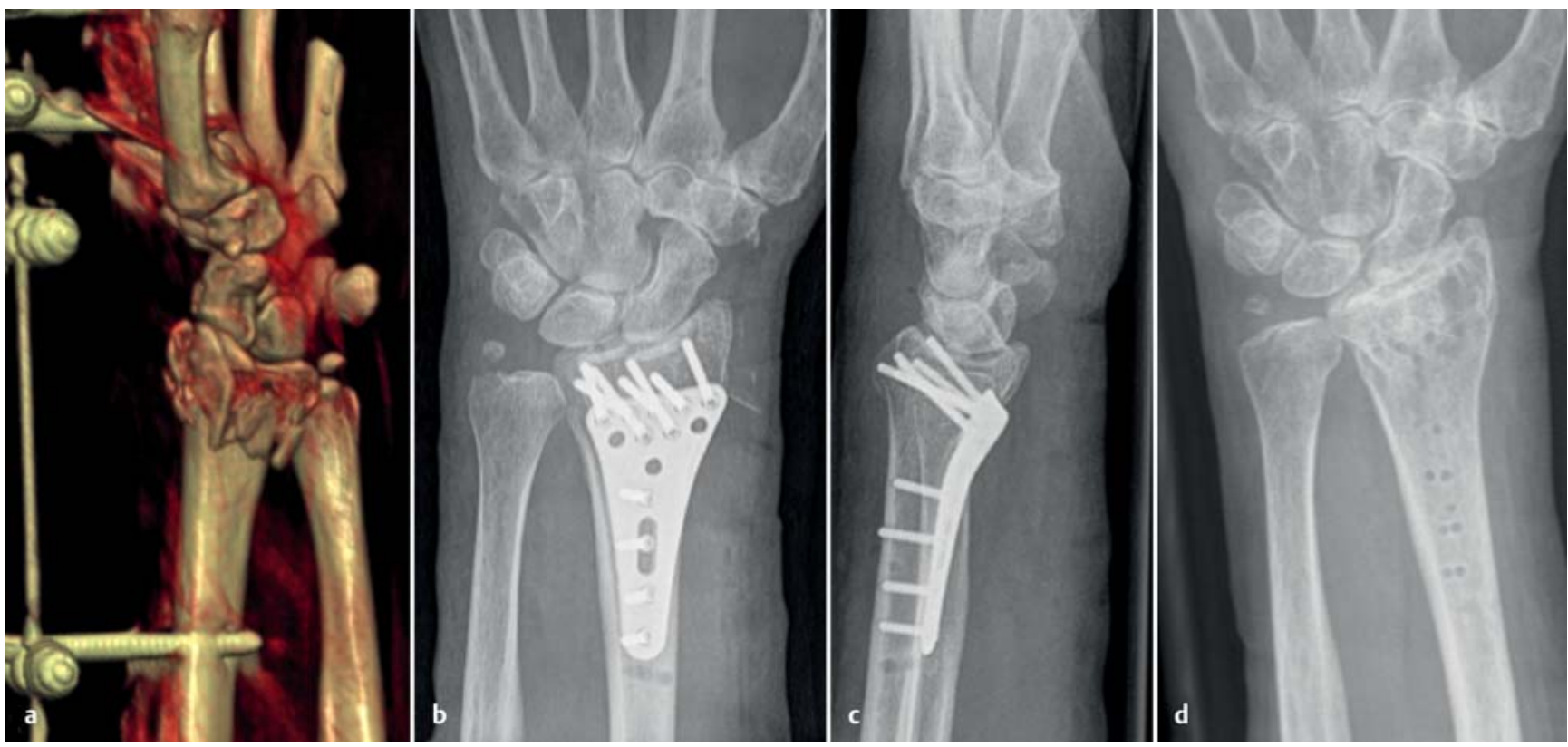

Abb. 1 a bis d Distale Radiusfraktur (AO23 C3) einer 78-jährigen ansonsten gesunden Patientin, Reposition und Plattenosteosynthese von palmarseitig bei guter Plattenlage und Frakturkonsolidierung. Z. n. komplikationsloser Implantatentfernung aufgrund von subjektiven Missempfindungen und Schmerzen.

Tab. 1 Risiken beim Belassen von Implantaten $[6,7]$ :

- Implantatbruch
- erschwerte Folgeeingriffe (insbeson-
dere Endoprothetik oder Arthrodese)
- Osteopenie durch „Stress Shielding“,
Schwächung des implantatgeschützten
Knochensegments
- Ermüdungsfraktur am Implantatende
- spätere bakterielle Besiedlung und
Implantatinfekt
- eingeschränkte Diagnostik (Artefakte)
- chronische Entzündung durch Korrosion

troffen, was eine IE deutlich erschweren kann. Diese Schwierigkeit ergibt sich insbesondere bei Implantaten, die bereits über einen längeren Zeitraum einliegen. Es sollte daher zur IE nach Möglichkeit ein gewisses Zeitfenster eingehalten werden. Wählt man den Zeitpunkt zu früh (vor abgeschlossener Frakturheilung) oder zu spät (nach knöchernem Ein- und Überwachsen des Implantats) riskiert man in beiden Fällen die Entstehung von Komplikationen, insbesondere von Refrakturen.

Mit Einführung winkelstabiler Plattensysteme ergab sich zudem das Problem des Metall-Metall-Verbunds, der sog. „Kaltverschweißung“ der Schraubenköpfe mit der Platte. Auch die Verwendung verschiedener Schraubenmitnahmeprofile (Kreuz, Schlitz, Stardrive, Inbus ${ }^{\circledR}$, Torx $\left.^{\circledR}\right)$ stellt zusätzlich hohe Anforderungen an das verwendete OP-
Instrumentarium. Derartige Schwierigkeiten können u.a. dazu führen, dass die Einbringung des Osteosynthesematerials elegant und minimalinvasiv erfolgt (bspw. mittels LISS [Less Invasive Stabilization System]), die Entfernung jedoch ein großer offener Eingriff wird, der den ursprünglich geplanten Aufwand deutlich übertrifft.

\section{Eingriffszahlen in Deutschland}

Vor diesem Hintergrund wird die Indikation zur Implantatentfernung heutzutage deutlich restriktiver gestellt. Dies spiegelt sich auch in den Zahlen von 2005 bis 2013 des Bundesamts für Statistik wider. Hier zeigt sich, dass die Anzahl der spezifischen OPS-Codes für die Osteosynthesematerialien Draht, Schraube, Zuggurtung, Platte und Nagel in dieser Zeit insgesamt um 7\% abgenommen hat. (2005: 118452 IE vs. 2013: 110123 IE). Diesem Abwärtstrend bei den IE steht ein Anstieg bei allen weiteren muskuloskeletalen Eingriffe gegenüber (+43,5\%). Die Abnahme der IE zeichnet sich jedoch nur bei den jüngeren Patienten ( $<65$ Jahre) ab (-13\%). Bei älteren Patienten ( $\geq 65$ Jahre) lässt sich in diesem Zeitraum sogar eine Zunahme (+ 7\%) verzeichnen.

Die Anzahl der Implantatentfernungen seit 2005 nimmt insgesamt ab (-7\%), insbesondere bei jüngeren Patienten $(-13 \%)$. Bei älteren Patienten ist jedoch eine Zunahme zu verzeichnen (+ 7\%).
Der demografische Wandel hat sicherlich einen Anteil an der Zunahme bei älteren Patienten. So stieg der Anteil der über 65-Jährigen in Deutschland allein in dem o.g. Zeitraum von 19 auf 21\% [8]. Naheliegend ist der Gedanke, dass die Zunahme der Entfernung von Osteosynthesematerial bei alten Patienten eine direkte Folge der Zunahme der Eingriffszahlen im Bereich der Endoprothetik sein könnte. Im Bereich von Knie und Hüfte sind die endoprothetischen Eingriffszahlen jedoch trotz alternder Bevölkerung seit 2009 kontinuierlich rückläufig.

Es deutet vieles darauf hin, dass ältere Menschen in Deutschland eine zunehmend längere Lebenserwartung in $\mathrm{Ge}$ sundheit haben. Es kommt daher wahrscheinlich eher zu einer Morbiditätskompression als zu einer -expansion [9]. Diese Entwicklung, die seit den 80erJahren beobachtet wird, könnte dazu beitragen, dass trotz des fortgeschrittenen Lebensalters, bei ansonsten fitten Patienten häufiger die Indikation zur (elektiven) IE gestellt wird.

Ältere Menschen in Deutschland haben eine zunehmend längere Lebenserwartung in Gesundheit, daher liegen seltener absolute Kontraindkationen zur Implantatentfernung vor.

Weiterhin zeigen die Daten des statistischen Bundesamts, dass im hohen Alter ein größerer Anteil an Frauen eine (elek- 


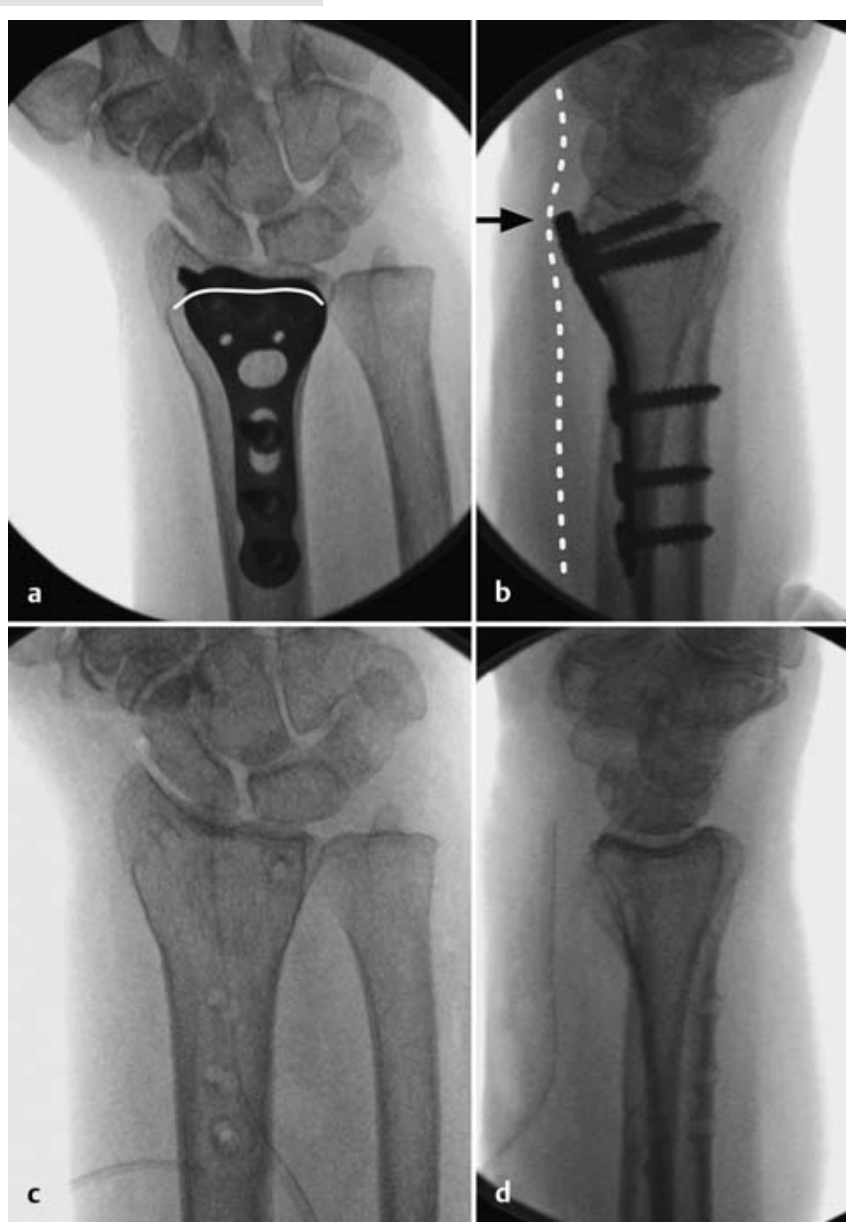

Abb. 2 a bis d Intraoperative Bildwandlerkontrolle: a, b zu weit distal gelegene Königssee-Platte bei konsolidierter distaler Radiusfraktur Handgelenk rechts p.-a. und lateral, schematische Darstellung der Watershed-Linie (weiße Linie), der Flexorensehnen (gestrichelt), Reibung über dem distalen Plattenende (Pfeil); c, d nach Implantatentfernung p.-a. und lateral. tive) IE vornehmen lässt. Bei den 65- bis 79-Jährigen beträgt das Verhältnis ca. $1: 3$ ( $\mathrm{m}: \mathrm{w})$, bei den über 80 -Jährigen ca. 1:5 (m: w). In unserer Klinik zeigt sich für die Jahre 2014-2015 bei den über 65-jährigen eine Geschlechtsverteilung von ca. 1:2 (m:w). Dieser hohe Anteil an Frauen liegt sicherlich auch darin begründet, dass Frauen im Alter etwa doppelt so häufig osteoporotische Frakturen erleiden, die eine Osteosynthese mit nachfolgender IE erfordern [10].

\section{Indikation zur Implantatentfernung}

Bei Infektionen, Pseudarthrosenbildung, Implantatversagen oder Weichteilschädigung herrscht Konsens darüber, dass die Indikation zur IE gegeben ist. Ebenso steht außer Frage, dass bei Kindern eine frühe elektive IE zur Verhinderung von Komplikationen des Knochenwachstums und zur Möglichkeit von weiteren korrigierenden Eingriffen, bspw. im Rahmen einer Hüftdysplasie, sinnvoll ist $[11,12]$. Die von vornherein zum Behandlungskonzept gehörende IE, wie z.B. die Stellschraubenentfernung am Sprunggelenk (mit Ausnahme gelockerter oder gebro- chener Stellschrauben), die K-Draht-Entfernung und die Entfernung von Provisorien, wie z.B. eines Fixateur externe, ist ebenso unbestritten sinnvoll und soll daher hier nicht vorrangiges Thema sein.

Bei jungen Erwachsenen (<40 Jahren) die nach Osteosynthese beschwerdefrei sind, ist eine routinemäßige IE nach Auffassung der meisten Orthopäden und Unfallchirurgen nicht unbedingt notwendig [1,11]. Die Meinungen gehen jedoch schon bei den relativen Indikationen deutlich auseinander. $\mathrm{Zu}$ diesen zählen u.a. Bewegungseinschränkung, Fremdkörpergefühl, Missempfindungen und die Einschränkung zukünftiger operativer Eingriffe, bspw. die Endoprothesenversorgung. Insbesondere der letzte Punkt spielt bei älteren Patienten eine große Rolle, da hier die Zeitpunkte zwischen Osteosynthese und Endoprothetik häufig nicht weit auseinander liegen.

Fortgeschrittenes Lebensalter gilt jedoch aktuell in den meisten Lehrbüchern aufgrund höherer peri- und postoperativer Risiken als Kontraindikation für die elektive Implantatentfernung. Im klinischen
Alltag gibt es allerdings immer wieder Grenzfälle, bei denen das Vorliegen von relativen Indikationen, wie z.B. implantatassoziierte Beschwerden und Bewegungseinschränkungen, auch beim alten Menschen für eine Implantatentfernung sprechen kann. In diesen Fällen muss eine besonders sorgfältige Abwägung des Risiko-Nutzen-Verhältnisses erfolgen.

Bei alten Patienten muss eine besonders sorgfältige Abwägung des Risiko-Nutzen-Verhältnisses erfolgen.

Derzeit ist die Indikationsstellung weitgehend von der individuellen Erfahrung des Chirurgen, den Gegebenheiten der Klinik oder regionalen Unterschieden abhängig. Auch Unterschiede in der Vergütung in verschiedenen Gesundheitssystemen können einen Einfluss auf die Häufigkeit der Durchführung einer IE haben. So liegt bspw. in Finnland die Rate an elektiven IE in großen Traumzentren bei bis zu $80 \%$, in den USA bei etwa $5 \%$, in Großbritannien bei $10 \%$, in den Niederlanden und in Deutschland bei 30 bis $40 \%$ [12].

Die Indikationsstellung zur Implantatentfernung ist derzeit weitgehend von der individuellen Erfahrung des Chirurgen, den Gegebenheiten der Klinik und regionalen Unterschieden in der Vergütung abhängig.

Eine Leitlinie zur IE liegt aktuell nur für Deutschland vor. Diese S1-Leitlinie „Implantatentfernung“ spiegelt die Kontroverse mit folgender Formulierung treffend wider: „Die Indikation zur Implantatentfernung ist relativ und selten zwingend. Sie wird immer individuell gestellt. Es gibt keine evidenzbasierte Empfehlung." [13]. Eine Implantatentfernung beim alten Menschen ist daher aufgrund des Risikoprofils eine besondere Herausforderung. Die Indikation muss gerade bei diesem Patientengut individuell mit Abwägen aller Nutzen und Risiken gestellt werden. Eine Übersicht über Indikationen zur IE beim alten Menschen wird in Tab. 2 vorgeschlagen.

\section{Obere Extremität - distaler Radius}

Implantate im Bereich der oberen Extremität sind im Gegensatz zur unteren Extremität im Alltag nicht so hohen statischen Belastungen ausgesetzt. Sie bewirken daher eine nicht so ausgeprägte Änderung der Biomechanik und können daher oft belassen werden. 
Tab. 2 Indikationen zur Implantatentfernung beim alten Menschen.

\begin{tabular}{lcl}
\hline klare Indikation & relative Indikation & keine Indikation \\
\hline - Schmerzen, Schwellungen und Irri- & - unklare Beschwer- & - Routine-IE oder Pa- \\
tationen im Bereich des Implantats & den ohne Schmer- & tientenwunsch bei \\
- funktionelle Einschränkungen & zen oder Funktions- & ansonsten beschwer- \\
- Wundheilungsstörungen und V.a. & einschränkungen & defreien Patienten \\
periimplantären Infekt & - V.a. auf Wundinfekt & - Osteosynthesematerial \\
- einzeitige IE und Wechsel auf En- & vor Konsolidierung & im Bereich von Hüfte \\
doprothese angrenzender Gelenke & der Fraktur & und Kniegelenk zur \\
- Implantatversagen (Ausnahme: & - vorderer Knie- & später einfacheren \\
gelockerte oder gebrochene Stell- & schmerz nach Tibia- & Endoprothetik \\
schrauben am Sprunggelenk) & nagelung & - Einschränkungen der \\
- periimplantäre Frakturen & - kosmetisch stören- & Diagnostik (MRT) \\
- Schrauben-„Cut-Out“ im Gelenk- & des Implantat & - Risiko der Korrosion, \\
bereich & & allergischer Reak- \\
- Femur- und Humeruskopfnekrose & & tionen und Kanzero- \\
- Sehnenrupturen (z.B dist. Radius) & & genese
\end{tabular}

Reizlos einliegende Implantate am distalen Radius mit freier Beweglichkeit im Handgelenk sollten belassen werden [14].

Plattenfehllagen am distalen Radius können jedoch chronische Sehnenschäden verursachen. Besonders häufig betroffen ist der M. extensor pollicis longus (EPL) und der M. flexor pollicis longus (FPL) [14]. Palmar überstehende Schrauben bzw. angebrachte Platten sind distal der "Watershed-Line“ nicht ausreichend durch den $M$. pronator quadratus gedeckt und können Schäden an Flexorensehnen verursachen. Folglich sollte ein Wechsel zu kürzeren Schrauben bzw. eine Entfernung der Platte oder ggf. eine Reosteosynthese erfolgen [14].

\section{Fallbeispiel 1: distaler Radius}

Die 78-jährige Patientin hatte nach einem Sturz auf die linke Hand eine mehrfragmentäre distale Radiusfraktur (AO23 C3) erlitten (Abb. 1). Nach initialer Fixierung mit einem Fixateur externe erfolgte die offene Reposition des distalen Radius, der radiokarpalen und ulnokarpalen Gelenkfläche und Osteosynthese mit einer 2,5-mm-Korrekturosteotomieplatte von palmarseitig. Eine Begleitfraktur des Processus styloideus ulnae (PSU) wurde nicht zusätzlich adressiert. Nach 6 Monaten stellte sich die Patientin erneut vor. Die Radiusfraktur war vollständig konsolidiert, die Narben und Weichteile zeigten sich trocken und reizlos. Die Beweglichkeit war in der klinischen Untersuchung gut. Die Platte trug in der klinischen Untersuchung nicht auf. Auch radiologisch gab es keinen Anhalt für eine Fehllage von Schrauben oder Platte. Dennoch berichtete die Patientin über gelegentliche Schmerzen und Missemp- findungen im Bereich des Implantats, daher wollte Sie unbedingt eine Entfernung des Implantats. Da bei der Patientin keine weiteren Vorerkrankungen vorlagen, die ein höheres Operationsrisiko bedeutet hätten, wurde nach ausführlicher Aufklärung die Indikation zur IE gestellt. Diese erfolgte komplikationslos. Die Patientin berichtete im Anschluss über eine Besserung der Schmerzsymptomatik. Anzumerken ist, dass in derartigen Fällen die relative Indikation des operativen Eingriffs im Rahmen der Aufklärung angesprochen werden muss und der Patient über die erhöhte Gefahr einer Refraktur informiert werden sollte.

Bei relativen OP-Indikationen muss besonders auf die Gefahr einer Refraktur hingewiesen werden.

\section{Fallbeispiel 2: distaler Radius}

Die auswärtig versorgte distale Radiusfraktur der 74-jährigen Patientin war nach 3 Jahren knöchern konsolidiert (Abb. 2). Beim Anheben eines Blumenkübels bemerkte die Patientin jedoch plötzlich ein schmerzhaftes „Knacken“ sowie ziehende Schmerzen im Handgelenk und Daumen. Eine Beugung im Interphalangealgelenk des Daumens war nicht mehr aktiv möglich. Die verwendete Königssee-Platte lag in diesem Fall $\mathrm{zu}$ weit distal und war nicht vom M. pronator quadratus überdeckt. Die stetige Reibung an der Platte bewirkte eine Ruptur der Sehne des M. flexor pollicis longus (FPL). Im Ultraschall zeigte sich der zurückgezogene Sehnenstumpf im Karpaltunnel. Es erfolgte daher die vollständige Entfernung der KönigsseePlatte und der Sehnenersatz der FPLSehne mit einem Streifen der Sehne des M. flexor carpi radialis (FCR). Im Verlauf zeigte sich eine wiederhergestellte Funktion der Daumenbeugung.

Beugesehnenrupturen im Bereich des Handgelenks sind eine Indikation zur Implantatentfernung.

\section{Schulter}

\section{Fallbeispiel 3: proximaler Humerus}

Die 69-jährige Patientin zog sich eine 3Part-Humeruskopffraktur zu, die mittels PHILOS-Platte und Anschlingen des Tuberculum majus (Ethibond ${ }^{\circledR} 6 / 0$ ) operativ versorgt wurde (Abb. 3 ). Nach Konsolidierung der Fraktur stellte sich die Patientin 1 Jahr später erneut vor. Insgesamt war die Patientin mit dem Operationsergebnis zufrieden, jedoch bemerke sie gelegentlich ziehende Schmerzen und eine Abduktionshemmung. Außerdem sei das Liegen auf der Seite schmerzhaft. Die Narbe und die Weichteile stellten sich reizlos und trocken dar. Die Abduktion war kraftvoll gegen Widerstand bis $90^{\circ}$ möglich. Die passive Mobilisation zeigte jedoch bei $120^{\circ}$ einen festen Anschlag. Außer einer latenten Hypothyreose und einer chronischen Gastritis war die Patientin ansonsten gesund. Nach ausführlicher Aufklärung wurde die Indikation zur IE gestellt. Intraoperativ war die Platte von derbem Narbengewebe überwachsen. Nach Freilegen der Platte konnte diese komplikationslos entfernt werden. In Narkose konnte die Schulter anschließend bis $160^{\circ}$ passiv abduziert werden. Die Patientin erhielt anschließend für 6 Wochen regelmäßig eine passive Mobilisation in einer Motorschiene. Mit dem Ergebnis ist die Patientin aktuell gut zufrieden, auch das Liegen auf der Seite bereite ihr keine Probleme mehr.

\section{Fallbeispiel 4: proximaler Humerus}

Der 90-jährige Patient stürzte im häuslichen Umfeld und erlitt eine subkapitale Humerusfraktur (A011-A2). Die offene Osteosynthese erfolgte mit einer kurzen PHILOS-Platte der Firma Synthes (Abb. 4). Postoperativ wurde 6 Wochen bei freier passiv-assistiver Beweglichkeit entlastet. An Vorerkrankungen bestanden ein Morbus Parkinson, eine arterielle Hypertonie, ein grauer Star, ein Basaliom und eine Presbyakusis. Vier Monate postoperativ erfolgte die Wiedervorstellung bei schmerzhafter Bewegungseinschränkung und Krepitationen bei Bewegung in der linken Schulter. Die Haut über dem OP-Gebiet zeigte sich reizlos und tro- 


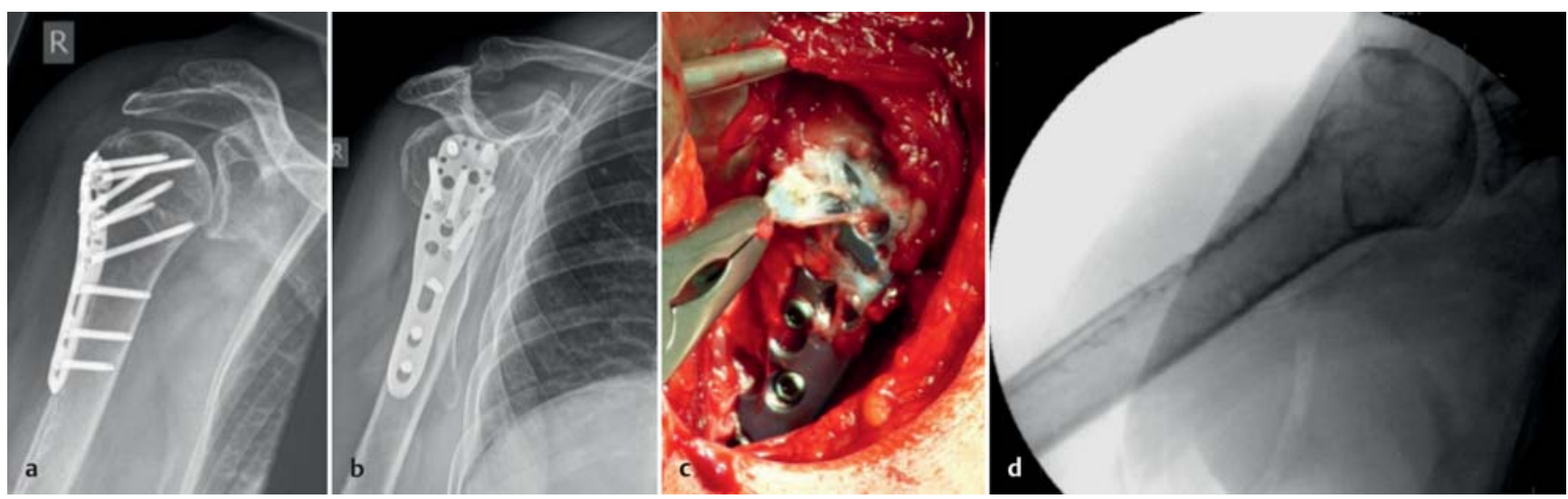

Abb. 3 a bis d Konsolidierte 3-Part-Humeruskopffraktur, 1 Jahr zuvor mit PHILOS-Platte versorgt, intraoperativer Befund, intraoperative Bildwandlerkontrolle.

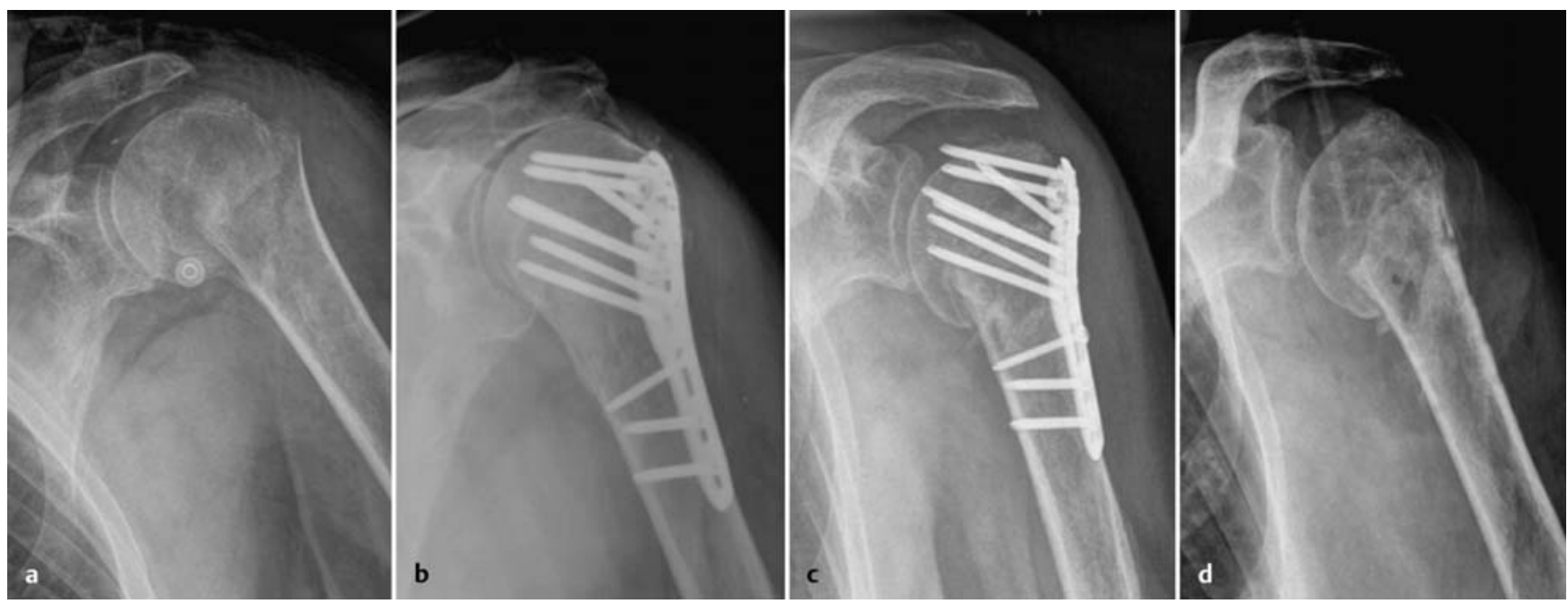

Abb. $\mathbf{4}$ a bis d Subkapitale Humerusfraktur bei einem 90-jährigen Patienten, Versorgung mittels kurzer PHILOS-Platte, intraartikuläre Schraubenlage nach Sinterung des Humeruskopfs 4 Monate postoperativ und Z. n. Implantatentfernung.

cken. Die periphere Durchblutung, Motorik und Sensibilität war intakt. Radiologisch zeigte sich eine zunehmende Sinterung und Einstauchung des Humeruskopfs mit intraartikulärer Schraubenfehllage. Der Frakturspalt war zunehmend konsolidiert, jedoch noch nicht vollständig durchbaut. Gemeinsam mit dem Patienten, der sich bis dato weitgehend selbstständig zu Hause versorgte, wurde die Entscheidung zur Implantatentfernung gestellt. Diese gelang komplikationslos, die Beweglichkeit im Schultergelenk ist seit dem Eingriff wieder beschwerdefrei möglich.

\section{Fallbeispiel 5: proximaler Humerus}

Der 73-jährige Patient erlitt eine mehrfragmentäre Humeruskopffraktur, die auswärts mit einer strahlendurchlässigen Karbonplatte operativ versorgt wurde (Abb.5). Bei zuvor bestehender adhäsiver Kapsulitis und AC-Gelenkar- throse hatte der Patient bereits mehrfach Injektionen eines Glukokortikoids in das Schultergelenk erhalten. Zudem war 3 Jahre zuvor eine Rotatorenmanschettennaht bei Partialruptur der Supraspinatussehne erfolgt. Als Vorerkrankung bestand ein Diabetes mellitus. Ein Jahr nach Osteosynthese stellte sich der Patient mit gelegentlich auftretenden ziehenden Schmerzen und Bewegungseinschränkung bei der Abduktion und Elevation vor. Beim Hausarzt seien intermittierend hohe Infektwerte aufgefallen. Im konventionellen Röntgen war der Frakturspalt medial nicht vollständig durchbaut und es bestanden Zeichen einer partiellen Implantatlockerung. Klinisch zeigte sich eine lokale Überwärmung und eine flächige, nicht juckende Effloreszenz über dem M. deltoideus. Bei V.a. eine Infektpseudarthrose wurde die Indikation zur Implantatentfernung gestellt.
Der Infekt konnte intraoperativ nicht nachgewiesen werden. Auch die entnommenen mikrobiologischen Proben zeigten kein Keimwachstum nach 7 Tagen. Jedoch bestand subdeltoidal direkt auf der Platte eine ca. $3 \times 8 \mathrm{~cm}$ große Pseudokapsel, aus der sich nach Eröffnung reichlich hypertrophes körniges Gewebe entleerte. Mit dem Finger konnte eine große Tasche getastet werden, welche bis hinter den Humeruskopf reichte. Dort bestand eine direkte Verbindung mit dem Gelenkraum. Histopathologisch wurde die Raumforderung als fibrosierte, pseudokapselartig gelagerte Synovialitis mit innen liegenden, knotigen und strangartigen Fibrinablagerungen ohne Anhalt für Malignität identifiziert. Nach vollständiger Entfernung der Pseudokapsel konnte schließlich auch die Platte vollständig entfernt werden. In der klinisch-radiologischen Verlaufskontrolle war der Patient mit der IE zufrieden und gab eine deutliche Schmerz- 

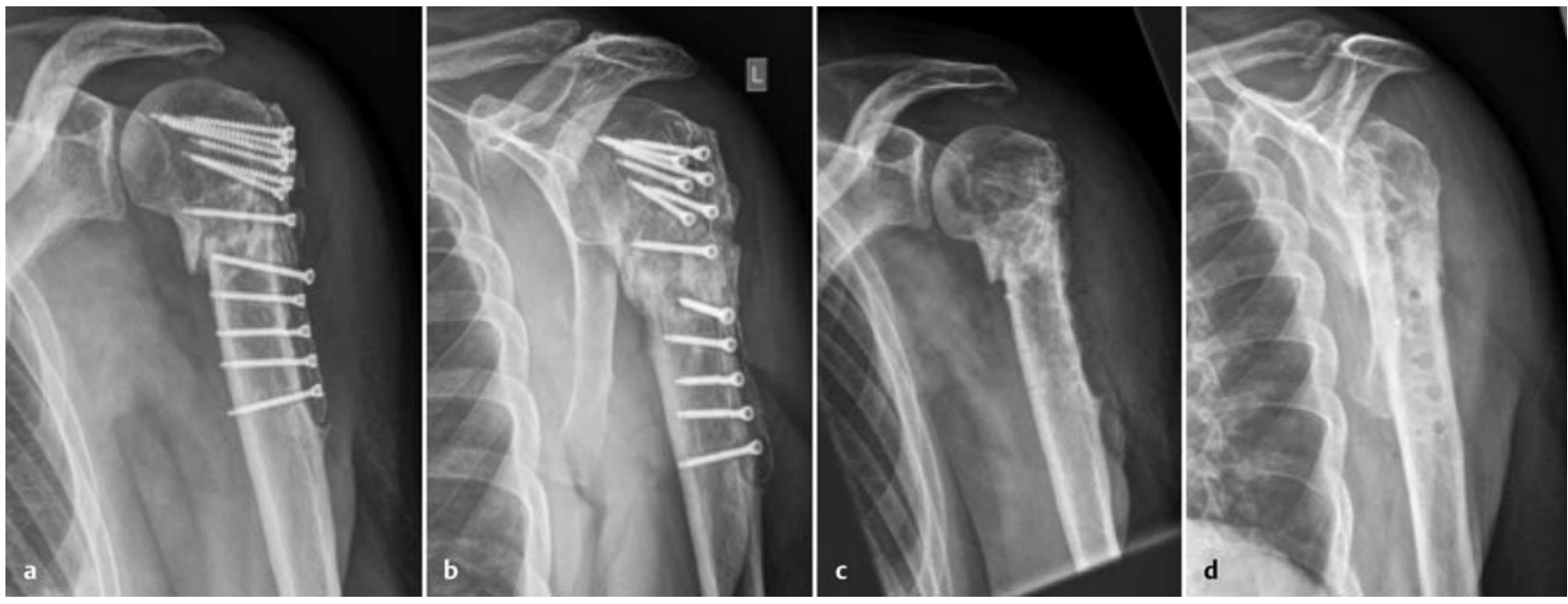

Abb. $\mathbf{5}$ a bis $\mathbf{d}$ Ein Jahr nach auswärtiger Versorgung einer nicht vollständig konsolidierten mehrfragmentären Humeruskopffraktur mit einer Karbonplatte, a.-p. und Y-View, Z. n. Implantatentfernung (IE), a.-p. und Y-View.
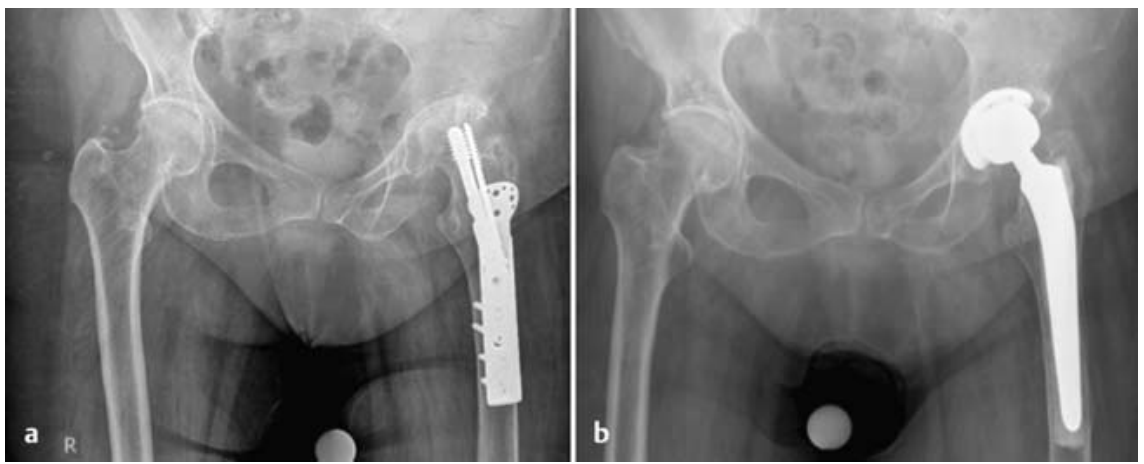

Abb. 6 a und b Fortgeschrittene Koxarthrose linkseitig 11 Jahre nach pertrochantärer Femurfraktur und Versorgung mit DHS und Trochanterabstützplatte, nach aufwendiger Implantatentfernung und Pfannenbodenplastik mit autologem Knochen Versorgung mittels Hybrid-Hüft-TEP rechts.

reduktion an. Auch der Frakturspalt zeigte sich nun zunehmend konsolidiert.

\section{Huifte}

Vielfach wird die Meinung geäußert, eine frühzeitige IE bei älteren Patienten im Bereich von Hüfte und Knie sei mit Blick auf die später wahrscheinlich notwendige endoprothetische Versorgung frühzeitig durchzuführen. Im Bereich des Hüftgelenks kann eine IE einer dynamischen Hüftschraube (DHS) jedoch durch den großen Substanzdefekt und Mikrofrakturierungen zu einer Schenkelhalsfraktur oder Hüftkopfnekrose führen [7]. Unserer Erfahrung nach lässt sich ein einzeitiger Wechsel auf eine Endoprothese auch bei jahrelang einliegendem Osteosynthesematerial durchführen und rechtfertigt nicht den zusätzlichen Eingriff einer frühzeitigen IE (siehe Fallbeispiel 6). In der aktuellen Literatur existieren hierzu jedoch noch keine Zahlen.
Eine frühzeitige elektive Entfernung von Osynthesematerial im Bereich der Hüfte ist beim alten Menschen im Hinblick auf eine später wahrscheinlich notwendige endoprothetische Versorgung allein nicht zu rechtfertigen.

\section{Fallbeispiel 6: proximales Femur}

Die 77-jährige Patientin klagte über progrediente Schmerzen und Einschränkung der Gehstrecke auf unter $50 \mathrm{~m}$ bei Koxarthrose links nach pertrochantärer Femurfraktur und Versorgung mit DHS und Trochanterabstützplatte vor 11 Jahren (Abb. 6). Es bestanden keine relevanten Vorerkrankungen. Aufgrund des langen Einliegens war das Osteosynthesematerial knöchern überbaut. Es ließ sich jedoch komplikationslos entfernen. Anschließend wurde eine Hybrid-Hüft-TEP mit Pfannenbodenplastik mit autolog gewonnenem Knochen implantiert. Es erfolgte eine orale Ossifkationsprophyla- xe mit Indometacin und eine orale Eisensubstitution. Nach 6 Monaten stellte sich die Patientin zur Verlaufskontrolle vor. Sie nahm keine Schmerzmittel mehr ein und konnte eine Gehstrecke von $300 \mathrm{~m}$ schmerzfrei gehen. In diesem Fall war der einzeitige Wechsel trotz des langen Einliegens der DHS gut möglich. Die frühzeitige Entfernung der DHS, lange vor der Versorgung mit einer Hüft-TEP, wäre hier nicht zu rechtfertigen gewesen.

\section{Proximale Tibia}

Vorderer Knieschmerz nach Marknagelung der Tibia beruht am ehesten auf einer Schädigung des Ramus infrapatellaris des N. saphenus [15]. Eine IE kann somit kaum eine Beschwerdelinderung bewirken. Im Gegenteil, es kann bei zuvor asymptomatischen Patienten eine Nervenläsion bei der IE auftreten [16]. Bei allen IE beim alten Menschen müssen die Erwartungen an den Erfolg einer IE kritisch geprüft und gegenüber den Risiken durch die erneute Operation abgewogen werden.

\section{Fallbeipiel 7: proximale Tibia}

Ein 65-jähriger Patient zog sich nach einem Motorradsturz im Jahr 2011 eine Tibiakopffraktur zu, die anschließend mittels LISS osteosynthetisch versorgt wurde (Abb. 7). Zwei Jahre später stellte er sich erneut mit Beschwerden im Bereich des rechten Knies vor. Das Auftragen des proximalen Plattenendes war deutlich durch die Haut sichtbar. Der Patient klagte über ständige Irritationen in dem Bereich und hatte einen großen OP-Wunsch. Obwohl die OP-Narbe reiz- 


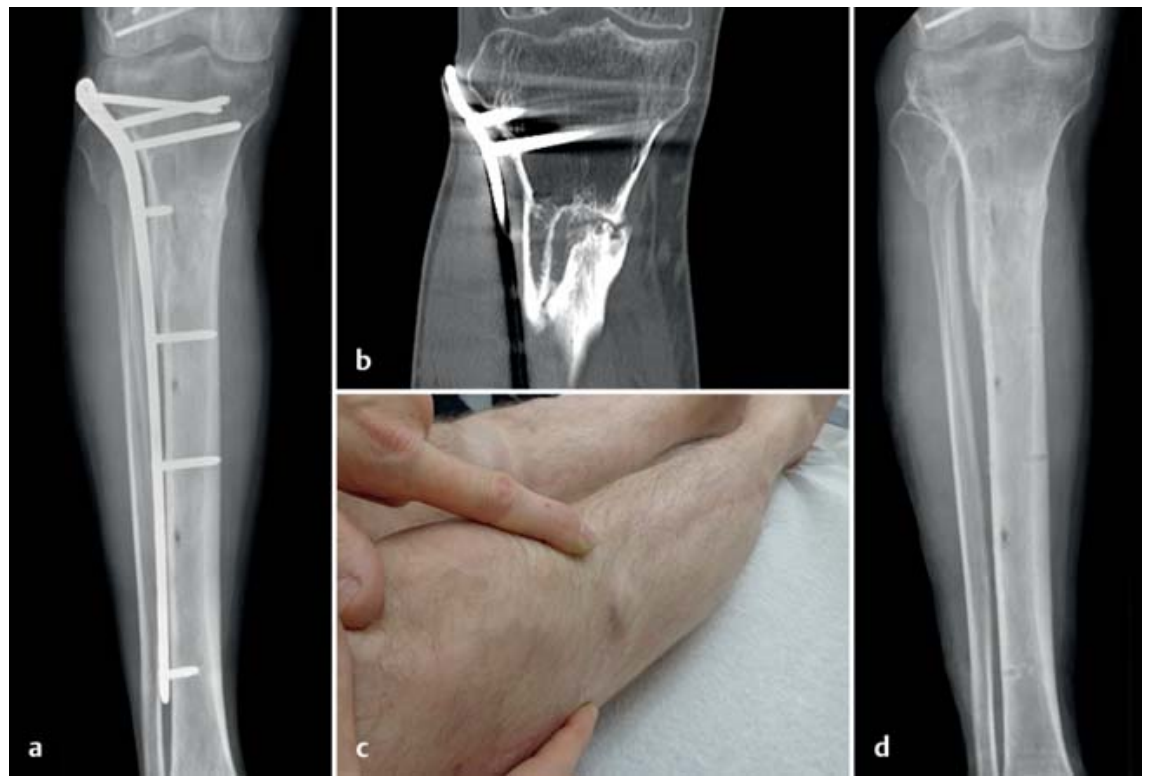

Abb.7a bis d Z.n. Tibiakopffraktur bei einem 65-Jährigen ohne sonstige Begleiterkrankungen, Hautirritation durch Auftragen einer LISS, komplikationslose Implantatentfernung.

los verheilt war, funktionell keine Einschränkungen bestanden und der Patient schon fortgeschrittenen Alters war, wurde eine Implantatentfernung bei relativer OP-Indikation durchgeführt. Begleiterkrankungen bestanden bei dem Mann nicht. Der erneute Eingriff gelang komplikationslos. Anschließend war der Patient beschwerdefrei.

\section{Sprunggelenk}

Implantate im Bereich des Sprunggelenks sollten nicht entfernt werden, sofern sie keine Beschwerden verursa- chen. Als Ausnahme gelten hier Stellschrauben, die routinemäßig vor Vollbelastung entfernt werden. Aufgrund der dünnen Weichteildeckung im Bereich des Außenknöchels kommt es jedoch häufig im Schuh zu Druckstellen. Gerade bei alten und multimorbiden Menschen, die aufgrund von Begleiterkrankungen, wie z.B. Diabetes mellitus, eine gestörte Sensorik im Bereich der Akren aufweisen, muss hierbei nicht nur auf eine Schmerzsymtomatik geachtet werden. Vielmehr sind hier eine schlechte Trophik der Haut und Weichteile, wie z. B. Indurationen, Ödeme, Ery- theme, kalte und livide Haut, zu beachten [17]. Durch eine Implantatentfernung im Bereich des Sprunggelenks kann bei symptomatischen Patienten häufig eine Besserung der Beschwerden erreicht werden. Jedoch darf der Erfolg der zusätzlichen Operation nicht überschätzt werden. Bei Pot et al. entwickelten insgesamt $43 \%(n=34)$ der Patienten nach ORIF (Open Reduction Internal Fixation) einer Sprunggelenksfraktur im Bereich der Implantate Schmerzen oder Missempfindungen [18]. Nach der Implantatentfernung erreichten zwar 71\% aller Patienten $(\mathrm{n}=80)$ nach 30 Monaten postoperativ eine deutliche Schmerzreduktion (NRS - Numerische RatingSkala) Mean: 7,0 vs. 3,9) [18]. Komplett schmerzfrei wurden jedoch nur $24 \%$ der Patienten und 29\% hatten weiterhin gleichbleibende Beschwerden.

Der Erfolg einer Implantatentfernung am Sprunggelenk zur Schmerzreduktion darf nicht überschätzt werden.

Hier gilt es, das individuelle Risiko der zusätzlichen Operation gegenüber dem potenziellen Nutzen abzuwägen. Die Patienten müssen speziell über die Aussicht auf Besserung ihrer Beschwerden aufgeklärt werden, um unrealistisch hohe Erwartungen an das Ergebnis auszuräumen. Für ältere Patienten im Speziellen ist die Datenlage diesbezüglich noch nicht ausreichend.
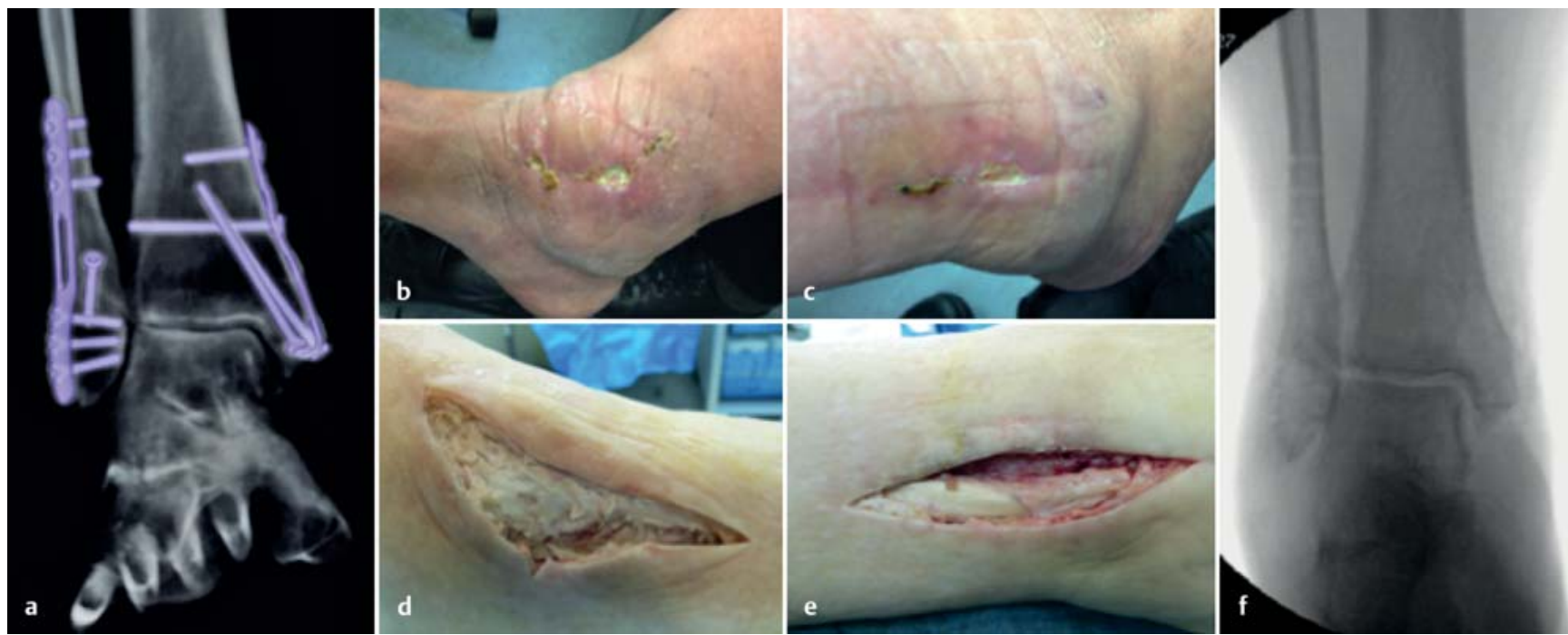

Abb. 8 a bis f VRT (Volume Rendering Technique): konsolidierte bimalleoläre Luxationsfraktur der 89-jährigen Patientin, Wundheilungsstörung im Bereich der medialen und lateralen OP-Narbe, intraoperativer Aspekt nach Wundrandausschneidung und Spülung, intraoperative Bildwandlerkontrolle nach vollständiger Implantatentfernung (IE). 


\section{Fallbeispiel 8: Sprunggelenk}

Die 89-jährige Patientin entwickelte 3 Monate nach Osteosynthese bei bimalleolärer Luxationsfraktur eine Wundheilungsstörung im Bereich der medialen und lateralen OP-Narben (Abb.8). An Vorerkrankungen bestanden ein Diabetes mellitus, eine Polyneuropathie der unteren Extremität und eine arterielle Hypertonie. Es bestand eine leichte Rötung ohne Überwärmung. Schmerzen hatte die Patientin aufgrund der Polyneuropathie im Bereich der OP-Narben nicht. Das CRP war geringfügig erhöht $(0,9 \mathrm{mg} / \mathrm{dl})$ bei normalen Leukozytenzahlen. Bei konsolidierter Fraktur und Verdacht auf einen Implantatinfekt erfolgte die IE der Konturenplatte, der Hakenplatte und der 2 freien 3,5-mmSchrauben. Nach Entnahme der mikrobiologischen Proben erfolgte ein ausgiebiges Débridement mit Wundspülung. Da eine spannungsfreie Primärnaht nach Wundrandausschneidung nicht möglich war, wurde eine Syspur-Derm ${ }^{\circledR}$-Weichschaumkompresse eingenäht. Bei Nachweis einer Mischinfektion mit Staphylococcus hominis, S. caprae und S. capitis und Corynebacterium amycolatum in den entnommenen Proben wurde eine resistogrammgerechte antibiotische Therapie begonnen. In der Folge wurde 2-malig revidiert und nach Anlage einer Dermotraktion am Außenknöchel eine spannungsfreie Sekundärnaht durchgeführt. Unter Fortführung der Antibiose heilten die OP-Narben reizlos ab. Die frühzeitige IE hat im vorliegenden Fall wahrscheinlich einen schweren Implantatinfekt mit knöcherner Beteiligung verhindert.

Der Verdacht auf einen implantatassoziierten Infekt ist i.d.R. eine klare Indikation zur sofortigen Implantatentfernung.

\section{Fazit}

Die Indikation zur Implantatentfernung kann nicht allein vom Alter der Patienten abhängig gemacht werden. Häufig kön- nen ältere Patienten ebenso wie jüngere Patienten von der IE profitieren. Jedoch müssen die Patienten über eine realistische Prognose aufgeklärt werden, um zu hohen Erwartungen an den Erfolg vorzubeugen. Eine IE ist aufgrund der Risiken des zusätzlichen operativen Eingriffs ausschließlich symptomatischen Patienten zu empfehlen. Bei unklaren Beschwerden ohne Schmerzen oder Funktionseinschränkungen sollte die Indikationsstellung sehr zurückhaltend erfolgen. Die frühzeitige IE bei beschwerdefreien älteren Patienten mit dem Ziel, einen möglichen späteren Eingriff zur Endoprothesenimplantation im Bereich von Knie, Hüfte und Schulter zu vereinfachen, ist aufgrund des Risikos des zusätzlichen Eingriffs nicht zu empfehlen. Ein einzeitiger Wechsel zu einer Endoprothese kann meist trotz lang einliegender Osteosynthesematerialien gut gelingen.

\section{Literatur}

${ }^{1}$ Hanson B, van der Werken C, Stengel D. Surgeons' beliefs and perceptions about removal of orthopaedic implants. BMC Musculoskelet Disord 2008; 9: 73

2 Bostman O, Pihlajamaki H. Routine implant removal after fracture surgery: a potentially reducible consumer of hospital resources in trauma units. The Journal of trauma 1996; 41: 846-849

${ }^{3}$ Müller M, Allgöwer M, Schneider R, Willenegger $H$. Manual of Internal Fixation, Techniques Recommended by the AO Group (translated by Shatzker J). Berlin, Heidelberg: Springer; 1979

${ }^{4}$ Grogan $\mathrm{CH}$. Experimental studies in metal cancerigenesis. VIII. On the etiological factor in chromate cancer. Cancer 1957; 10: 625638

${ }^{5}$ Levine D, Staehle R. Crevice corrosion in orthopedic implant metals. Journal of biomedical materials research 1977; 11: 553-561

${ }^{6}$ Wirth CJ, Mutschler W-E, Bischoff H-P, Püschmann $\mathrm{H}$, Neu J. Komplikationen in Orthopädie und Unfallchirurgie: vermeiden - erkennen behandeln: Stuttgart: Thieme; 2009

7 Müller-Färber J. Die Metallentfernung nach Osteosynthesen. Indikationen und Risiken Orthopäde 2003; 32: 1039-1057.

${ }^{8}$ DESTATIS Statistisches Bundesamt Bevölkerung in Deutschland. [updated 10.01.2016]. Im Internet: https://http://www.destatis.de/ bevoelkerungspyramide $/-! \mathrm{y}=2013 \& \mathrm{~V}=2 \& 0=$ 2013\&g.; Stand: 10.01.2016
${ }^{9}$ Böhm K, Tesch-Römer C, Ziese T. Gesundheit und Krankheit im Alter - Beiträge zur Gesundheitsberichterstattung des Bundes. Berlin: Robert Koch-Institut; 2009

${ }^{10}$ Kanis J, Burlet N, Cooper C et al. European guidance for the diagnosis and management of osteoporosis in postmenopausal women. Osteoporosis international 2008; 19: 399428

11 Jamil W, Allami M, Choudhury M et al. Do orthopaedic surgeons need a policy on the removal of metalwork? A descriptive national survey of practicing surgeons in the United Kingdom. Injury 2008; 39: 362-367

12 Vos D, Hanson B, Verhofstad M. Implant removal of osteosynthesis: the Dutch practice. Results of a survey. Journal of Trauma Management \& Outcomes 2012; 6: 6

${ }^{13}$ Jürgen MIF. Leitlinien Unfallchirurgie überarbeitete Leitlinie S1- Implantatentfernung. Leitlinienkommission der Deutschen Gesellschaft für Unfallchirurgie eV (DGU): 2014

${ }^{14}$ Krettek C, Müller C, Meller $R$ et al. [Is routine implant removal after trauma surgery sensible?]. Unfallchirurg 2012; 115: 315-322

15 Leliveld MS, Verhofstad MH. Injury to the infrapatellar branch of the saphenous nerve, a possible cause for anterior knee pain after tibial nailing? Injury $2012 \cdot 43: 779-783$

${ }^{16}$ Karladani AH, Ericsson PA, Granhed $\mathrm{H}$ et al. Tibial intramedullary nails - should they be removed? A retrospective study of 71 patients. Acta orthopaedica 2007; 78: 668-671

17 Ochman S, Evers J, Raschke MJ. Sprunggelenksfraktur im Alter - was ist anders? OPJOURNAL 2014; 30: 76-80

18 Pot J, van Wensen R, Olsman J. Hardware related pain and hardware removal after open reduction and internal fixation of ankle fractures. The Foot and Ankle Online Journal 2011; 4(5): 1

Dr. med. Jan Pützler M.Sc. Assistenzarzt

PD Dr. med. Richard Stange Leitender Oberarzt Dr. med. Christoph Domnick Assistenzarzt

Univ.-Prof. Michael J. Raschke

Klinikdirektor

Klinik und Poliklinik für Unfall-, Handund Wiederherstellungschirurgie

Universitätsklinikum Münster

Albert-Schweitzer-Campus 1

48149 Münster

Michael.Raschke@ukmuenster.de 